OPEN ACCESS

Edited by:

Johnny Kao,

Good Samaritan Hospital Medical

Center, United States

Reviewed by:

Amar Kishan,

University of California, Los Angeles,

United States

Angela G. M. O'Neill,

Belfast Health and Social Care Trust,

United Kingdom

*Correspondence:

Jonathan Haas

jonathan.haas@nyulangone.org

Specialty section:

This article was submitted to

Radiation Oncology,

a section of the journal

Frontiers in Oncology

Received: 12 October 2019

Accepted: 06 February 2020

Published: 27 February 2020

Citation:

locolano M, Blacksburg S,

Carpenter T, Repka M, Carbone S,

Demircioglu G, Miccio M, Katz A and

Haas J (2020) Prostate Fiducial

Marker Placement in Patients on

Anticoagulation: Feasibility Prior to Prostate SBRT. Front. Oncol. 10:203.

doi: 10.3389/fonc.2020.00203

\section{Prostate Fiducial Marker Placement in Patients on Anticoagulation: Feasibility Prior to Prostate SBRT}

\author{
Michelle locolano ${ }^{1,2}$, Seth Blacksburg ${ }^{1}$, Todd Carpenter ${ }^{1}$, Michael Repka ${ }^{1}$, \\ Susan Carbone ${ }^{1}$, Gizem Demircioglu ${ }^{1}$, Maryann Miccio ${ }^{1}$, Aaron Katz ${ }^{3}$ and \\ Jonathan Haas ${ }^{1 *}$ \\ ${ }^{1}$ Department of Radiation Oncology, NYU Winthrop Hospital, Mineola, NY, United States, ${ }^{2}$ Stony Brook University School of \\ Medicine, Stony Brook, NY, United States, ${ }^{3}$ Department of Urology, NYU Winthrop Hospital, Mineola, NY, United States
}

Background and Purpose: Fiducial marker placement is required in patients undergoing robotic-based Stereotactic Body Radiotherapy (SBRT) or image-guided radiation therapy (IGRT) for prostate cancer. Many patients take antiplatelet or anticoagulant medication due to other medical comorbidities. They are often required to temporarily discontinue these medications prior to invasive medical procedures as they are prone to bleed. Some patients are unable to discontinue therapy due to an elevated risk of thromboembolic events. The purpose of this study is to report this institution's experience placing fiducial markers in prostate cancer patients who are on chronic antiplatelet or anticoagulant medication.

Materials and Methods: From August 2015-March 201957 patients on chronic antiplatelet or anticoagulation therapy who were not cleared to stop these medications underwent transrectal ultrasound guided (TRUS) fiducial marker placement for SBRT/IGRT. All patients were monitored by a registered nurse during the procedure for prolonged bleeding that required staff to hold pressure to the area with a $4 \times 4$ gauze until it resolved. All patients were also called the following day to assess for ongoing bleeding events. Treatment planning CT scan confirmed the ideal geometry of the marker placement.

Results: All 57 patients on antiplatelet or anticoagulant medication who underwent fiducial marker placement were discharged home the same day of the procedure. Four patients experienced persistent bleeding that required a nurse to hold prolonged pressure to the area. No patient experienced significant bleeding the following day or any untoward cardiovascular event.

Conclusions: This series suggests the use of antiplatelet or anticoagulant medication is not an absolute contraindication to fiducial marker placement in patients undergoing SBRT or IGRT for prostate cancer. These patients should be closely monitored after the 
procedure for bleeding complications. Practitioners may consider the patient's medical comorbidities, risk factors for thromboembolism, and overall functional status as there is no standardized protocol for discontinuing anticoagulant or antiplatelet therapy for fiducial marker placement.

Keywords: fiducial markers, radiotherapy, image-guided, anticoagulants, prostate cancer, SBRT

\section{INTRODUCTION}

In 2019, it is anticipated that prostate cancer will account for almost 20 percent of new cancer diagnoses in men (1). There are many options for treating localized prostate cancer. Stereotactic body radiation therapy (SBRT) allows precise delivery of a large dose of radiation to the prostate in one to five fractions with toxicity rates comparable to conventionally fractionated radiation (2-5). Placement of fiducial markers prior to radiotherapy is necessary for robotic-based SBRT to account for the six degrees of prostate motion during treatment and to prevent over- irradiation of normal surrounding tissue $(6,7)$. Fiducials are utilized in the majority of those treated with SBRT delivered with gantry-mounted linear accelerators although their role in platforms equipped with cone beam CT imaging is actively being debated (8).

Many patients with prostate cancer eligible for SBRT are on chronic antiplatelet or anticoagulant medication for other medical comorbidities. Fiducial marker placement is an invasive procedure that confers an elevated risk for bleeding complications in these patients and several institutions require the temporary discontinuation of their medication prior to the procedure $(6,7,9)$. For many patients, however, this is not feasible due an elevated risk of thromboembolic events. One potential alternative for patients who require active anticoagulation being treated on a unit with cone beam CT are prostatic calculi as they are prevalent in $85 \%$ of patients (10). To our knowledge, no study has assessed the feasibility of maintaining antiplatelet or anticoagulant medication prior to fiducial marker placement. The purpose of this study is to report a high-volume academic institution's experience placing fiducial markers for prostate SBRT in this patient population.

\section{MATERIALS AND METHODS}

\section{Patient Population}

This study was approved by the institutional review board at NYU Winthrop Hospital. From August 2015-March 2019, 57 patients with prostate cancer scheduled for fiducial marker placement and subsequent SBRT or image guided radiation therapy (IGRT) at NYU Winthrop Hospital were identified and consented to the study. Eligible patients included those on chronic antiplatelet or anticoagulant medication who were not cleared to stop their medication prior to fiducial marker placement. Antiplatelet medications included aspirin and clopidogrel. Anticoagulants included warfarin, heparin, apixaban, rivaroxaban, and dabigatran. There was no exclusion criteria and no patients declined to undergo fiducial marker placement. Fiducials were placed by three radiation oncologists. Nursing staff monitored all patients during the procedure to assess for prolonged bleeding from the perineum which required the use of sustained pressure to the area with a $4 \times 4$ gauze until it resolved. All patients were also called 1 day after the procedure to assess for persistent bleeding events.

\section{Treatment Technique}

Study participants had four gold fiducial markers placed in the prostate transperineally with transrectal ultrasound guidance prior to SBRT/IGRT. Prior to marker placement, lidocaine gel, and EMLA cream were used to numb the perineum and rectum. Two needles were double loaded with two gold fiducial markers each with a spacer in between. The needles were inserted transperineally into the prostate. Two fiducial seeds were placed at the right and left base and two seeds were placed in the right and left apex. Following withdrawal of the ultrasound transducer and needle, nursing staff provided gentle pressure to the area and monitored patients for persistent bleeding. A treatment planning CT scan was used to confirm the ideal geometry of the marker placement. Patients were planned and treated as previously described (11).

\section{RESULTS}

The average age of the study population was 70.3 with a range of 54-83. All 57 patients who underwent fiducial marker placement were on antiplatelets or anticoagulation and discharged home on the same day of the procedure. Baseline patient characteristics are found in Table 1. As seen in Table 2, most patients were on a single antiplatelet agent $(63.2 \%)$, followed by a single anticoagulant (17.5\%), dual antiplatelet agents (17.5\%), and triple anticoagulant/antiplatelet agents $(1.8 \%)$ at the time of procedure. Medical comorbidities requiring the use of anticoagulation or antiplatelet medication are listed in Table 3. Coronary artery disease $(56.1 \%)$ and primary prevention $(17.5 \%)$ were most commonly reported.

Four patients experienced transient CTCAE grade 1 bleeding at the time of fiducial placement requiring a registered nurse to provide sustained pressure on the perineum with a $4 \times 4$ gauze. Two of these patients took Aspirin $81 \mathrm{mg}$ daily; one took Aspirin $162 \mathrm{mg}$ daily; and one patient took Aspirin $81 \mathrm{mg}$, Eliquis $5 \mathrm{mg}$, and Plavix $75 \mathrm{mg}$ daily. The following day, these patients reported via telephone they did not experience further bleeding events. No other significant bleeding or cardiovascular events in the periprocedural window were reported. There were no cases of fiducial remediation in this cohort and all patients subsequently received definitive SBRT for their prostate malignancy without untoward 
TABLE 1 | Baseline patient characteristics.

\begin{tabular}{lcc}
\hline & $\boldsymbol{n}=$ & (\%) \\
\hline Clinical stage & 3 & \\
Tx & 43 & 5.3 \\
T1c & 2 & 75.4 \\
T2a & 5 & 3.5 \\
T2b & 1 & 8.8 \\
T2C & 3 & 1.8 \\
T3a & 0 & 5.3 \\
T3b & Mean 9.08 ng/ml (3.0-48.0) & \\
PSA & 39 & 08.4 \\
$<10 \mathrm{ng} / \mathrm{ml}$ & 16 & 28.1 \\
10-20 ng/ml & 2 & 3.5 \\
$>$ 20 ng/ml & & \\
Gleason score & 10 & 17.5 \\
6 (3+3) & 21 & 36.8 \\
7 (3+4) & 14 & 24.6 \\
7 (4+3) & 12 & 21.1 \\
8-10 & & \\
NCCN risk stratification & 7 & 63.2 \\
Low & 36 & 24.6 \\
Intermediate & 14 & \\
High & & \\
\hline
\end{tabular}

medical event precluding completion of their prostate cancer treatment. No patient had signs or symptoms to indicate further bleeding on initiation of radiation treatment.

\section{DISCUSSION}

SBRT offers patients with low- to intermediate risk prostate cancer the convenience of a shortened treatment course with excellent local control rates and treatment toxicities comparable to other definitive radiation modalities. Patients who opt for fiducial marker placement and subsequent SBRT may also be on antiplatelet or anticoagulant regimens for other medical conditions. A review of the literature suggests patients may be required to temporarily discontinue their medication prior to fiducial placement $(6,7,9,12)$. For some patients this is not possible due to an elevated risk of thromboembolism. There is limited evidence to guide management in these patients with a theoretically increased risk of bleeding following fiducial marker placement.

At our institution, 57 patients on chronic antiplatelet or anticoagulant medication were not found to have significant bleeding events following the placement of fiducial markers for prostate SBRT. While four patients experienced bleeding at the time of procedure, it had resolved with no further complications as per patient report the following day. Similarly, Ihezue et al. suggest that patients on warfarin did not experience clinically significant hematuria, hematospermia or rectal bleeding within 10 days following transrectal ultrasound guided prostate biopsy when compared to those not on anticoagulants (13). While
TABLE 2 | Antiplatelet and anticoagulant medication.

\begin{tabular}{|c|c|c|}
\hline & $n=$ & (\%) \\
\hline \multicolumn{3}{|l|}{ Single agent } \\
\hline \multicolumn{3}{|l|}{ Antiplatelet } \\
\hline Aspirin & 28 & 49.1 \\
\hline Clopidogrel & 8 & 14.0 \\
\hline Total & 36 & 63.2 \\
\hline \multicolumn{3}{|l|}{ Anticoagulant } \\
\hline Warfarin & 3 & 5.3 \\
\hline Heparin & 3 & 5.3 \\
\hline Apixaban & 1 & 1.8 \\
\hline Rivaroxaban & 2 & 13.5 \\
\hline Dabigatran & 1 & 1.8 \\
\hline Total & 10 & 17.5 \\
\hline \multicolumn{3}{|l|}{ Dual agent } \\
\hline $\begin{array}{l}\text { Aspirin + } \\
\text { Plavix }\end{array}$ & 6 & 1.5 \\
\hline $\begin{array}{l}\text { Apixaban + } \\
\text { Plavix }\end{array}$ & 4 & 7.0 \\
\hline Total & 10 & 17.5 \\
\hline \multicolumn{3}{|l|}{ Three agents } \\
\hline $\begin{array}{l}\text { Aspirin + } \\
\text { Apixaban + } \\
\text { Clopidogrel }\end{array}$ & 1 & 1.8 \\
\hline Total & 1 & 1.8 \\
\hline
\end{tabular}

TABLE 3 | Patient comorbidities requiring use of anticoagulant or antiplatelets.

\begin{tabular}{lcr}
\hline & $\boldsymbol{n}=$ & (\%) \\
\hline Pulmonary embolism & 2 & 3.5 \\
Coronary artery disease & 32 & 56.1 \\
Atrial Fibrillation & 3 & 5.3 \\
Cerebral vascular accident & 2 & 3.5 \\
Primary prevention & 10 & 17.5 \\
Other & 3 & 8.8 \\
Unknown & 5 & 5.3 \\
\hline
\end{tabular}

Saito et al. analogously found comparable bleeding risk in patients on anticoagulant or antiplatelet therapy and those who were not, patients on these medications were more likely to have clot retention and minor bleeding following biopsy. Notably, there was no report of significant bleeding events in any patient undergoing transrectal ultrasound guided biopsy $(13,14)$.

While this is the first study to suggest that chronic antiplatelet or anticoagulant medication is not an absolute contraindication for seed placement, further research is required. The small sample size in this study may be insufficient to detect bleeding complications in the larger patient population eligible for this procedure and a prospective, randomized study with greater patient numbers could provide greater clarity on this topic. While there is no standardized protocol for discontinuing these medications in preparation for fiducial marker placement, 
practitioners must consider many clinical factors including overall risk of thromboembolism, functional status, other comorbidities prior to making a treatment decision.

\section{DATA AVAILABILITY STATEMENT}

All datasets generated for this study are included in the article/supplementary material.

\section{ETHICS STATEMENT}

The studies involving human participants were reviewed and approved by NYU Winthrop Hospital IRB. The

\section{REFERENCES}

1. Siegel RL, Miller KD, Jemal A. Cancer statistics, 2019. CA Cancer J Clin. (2019) 69:7-34. doi: 10.3322/caac.21551

2. Boyer MJ, Papagikos MA, Kiteley R, Vujaskovic Z, Wu J, Robert Lee W. Toxicity and quality of life report of a phase II study of stereotactic body radiotherapy (SBRT) for low and intermediate risk prostate cancer. Radiat Oncol. (2017) 12:14. doi: 10.1186/s13014-016-0758-8

3. Widmark A, Gunnlaugsson A, Beckman L, Thellenberg-Karlsson C, Hoyer M, Lagerlund $\mathrm{M}$, et al. Ultra-hypofractionated versus conventionally fractionated radiotherapy for prostate cancer: 5-year outcomes of the HYPO-RTPC randomised, non-inferiority, phase 3 trial. Lancet. (2019) 394:38595. doi: 10.1016/S0140-6736(19)31131-6

4. Kishan AU, Dang A, Katz AJ, Mantz CA, Collins SP, Aghdam N, et al. Long-term outcomes of stereotactic body radiotherapy for lowrisk and intermediate-risk prostate cancer. JAMA Netw Open. (2019) 2:e188006. doi: 10.1001/jamanetworkopen.2018.8006

5. Brand DH, Tree AC, Ostler P, van der Voet H, Loblaw A, Chu W, et al. Intensity-modulated fractionated radiotherapy versus stereotactic body radiotherapy for prostate cancer (PACE-B):acute toxicity findings from an international, randomised, open-label, phase 3, non-inferiority trial. Lancet Oncol. (2019) 20:1531-43. doi: 10.1016/S1470-2045(19)30569-8

6. Saad A, Goldstein J, Lawrence YR, Weiss I, Saad R, Spieler B, et al. Transperineal implantation of gold fiducial markers (gold seeds) for prostate image-guided radiation therapy: a feasible technique associated with a low risk of complications. J Med Radiat Sci. (2015) 62:261-6. doi: 10.1002/j mrs. 122

7. Gill S, Li J, Thomas J, Bressel M, Thursky K, Styles C, et al. Patientreported complications from fiducial marker implantation for prostate imageguided radiotherapy. $\mathrm{Br} J$ Radiol. (2012). 85:1011-7. doi: 10.1259/bjr/681 27917

8. Njeh CF, Parker BC, Orton CG. Implanted fiducial markers are no longer needed for prostate cancer radiotherapy. Med Phys. (2017) 44:61136. doi: $10.1002 / \mathrm{mp} .12633$ patients/participants provided their written informed consent to participate in this study.

\section{AUTHOR CONTRIBUTIONS}

$\mathrm{MI}$ and $\mathrm{JH}$ were responsible for the conception of this study. All authors contributed to the writing of this review, editing, and final approval prior to submission.

\section{FUNDING}

$\mathrm{JH}$ and SB have received honoraria from Accuray.

9. Fawaz ZS, Yassa M, Nguyen DH, Vavassis P. Fiducial marker implantation in prostate radiation therapy: complication rates and technique. Cancer Radiother. (2014). 18:736-9. doi: 10.1016/j.canrad.2014.07.160

10. O'Neill AGM, Osman SO, Jain S, Hounsell AR, O'Sullivan JM. Observed high incidence of prostatic calculi with the potential to act as natural fiducials for prostate image guided radiotherapy. Tech Innov Patient Suport Radiat Oncol. (2019) 9:35-40. doi: 10.1016/j.tipsro.2019.01.004

11. Vu CC, Haas JA, Katz AE, Witten MR. Prostate-specific antigen bounce following stereotactic body radiation therapy for prostate cancer. Front Oncol. (2014). 4:8. doi: 10.3389/fonc.2014.00008

12. Kably I, Bordegaray M, Shah K, Salsamendi J, Narayanan G. Single-center experience in prostate fiducial marker placement: technique and midterm follow-up. J Vasc Interv Radiol. (2014). 25:1125-32. doi: 10.1016/j.jvir.2014.03.017

13. Ihezue CU, Smart J, Dewbury KC, Mehta R, Burgess L. Biopsy of the prostate guided by transrectal ultrasound: relation between warfarin use and incidence of bleeding complications. Clin Radiol. (2005). 60:459-63; discussion: 4578. doi: 10.1016/j.crad.2004.10.014

14. Saito K, Washino S, Nakamura Y, Konishi T, Ohshima M, Arai Y, et al. Transperineal ultrasound-guided prostate biopsy is safe even when patients are on combination antiplatelet and/or anticoagulation therapy. BMC Urol. (2017). 17:53. doi: 10.1186/s12894-017-0245-Z

Conflict of Interest: The authors declare that the research was conducted in the absence of any commercial or financial relationships that could be construed as a potential conflict of interest.

Copyright (c) 2020 Iocolano, Blacksburg, Carpenter, Repka, Carbone, Demircioglu, Miccio, Katz and Haas. This is an open-access article distributed under the terms of the Creative Commons Attribution License (CC BY). The use, distribution or reproduction in other forums is permitted, provided the original author(s) and the copyright owner(s) are credited and that the original publication in this journal is cited, in accordance with accepted academic practice. No use, distribution or reproduction is permitted which does not comply with these terms. 